\title{
A new assay for measuring chromosome instability (CIN) and identification of drugs that elevate CIN in cancer cells
}

Hee-Sheung Lee ${ }^{1}$, Nicholas CO Lee ${ }^{1}$, Brenda R Grimes², Alexander Samoshkin', Artem V Kononenko', Ruchi Bansal ${ }^{2}$, Hiroshi Masumoto ${ }^{3}$, William C Earnshaw ${ }^{4}$, Natalay Kouprina ${ }^{1}$ and Vladimir Larionov ${ }^{1 *}$

\begin{abstract}
Background: Aneuploidy is a feature of most cancer cells that is often accompanied by an elevated rate of chromosome mis-segregation termed chromosome instability (CIN). While CIN can act as a driver of cancer genome evolution and tumor progression, recent findings point to the existence of a threshold level beyond which CIN becomes a barrier to tumor growth and therefore can be exploited therapeutically. Drugs known to increase CIN beyond the therapeutic threshold are currently few in number, and the clinical promise of targeting the CIN phenotype warrants new screening efforts. However, none of the existing methods, including the in vitro micronuclei (MNi) assay, developed to quantify CIN, is entirely satisfactory.

Methods: We have developed a new assay for measuring CIN. This quantitative assay for chromosome missegregation is based on the use of a non-essential human artificial chromosome (HAC) carrying a constitutively expressed EGFP transgene. Thus, cells that inherit the HAC display green fluorescence, while cells lacking the HAC do not. This allows the measurement of HAC loss rate by routine flow cytometry.

Results: Using the HAC-based chromosome loss assay, we have analyzed several well-known anti-mitotic, spindletargeting compounds, all of which have been reported to induce micronuclei formation and chromosome loss. For each drug, the rate of HAC loss was accurately measured by flow cytometry as a proportion of non-fluorescent cells in the cell population which was verified by FISH analysis. Based on our estimates, despite their similar cytotoxicity, the analyzed drugs affect the rates of HAC mis-segregation during mitotic divisions differently. The highest rate of HAC mis-segregation was observed for the microtubule-stabilizing drugs, taxol and peloruside A.

Conclusion: Thus, this new and simple assay allows for a quick and efficient screen of hundreds of drugs to identify those affecting chromosome mis-segregation. It also allows ranking of compounds with the same or similar mechanism of action based on their effect on the rate of chromosome loss. The identification of new compounds that increase chromosome mis-segregation rates should expedite the development of new therapeutic strategies to target the CIN phenotype in cancer cells.
\end{abstract}

Keywords: Human artificial chromosome, HAC, Chromosome instability, CIN, Drug treatment

\footnotetext{
* Correspondence: larionov@mail.nih.gov

'Laboratory of Molecular Pharmacology, National Cancer Institute, Bethesda, MD 20892, USA

Full list of author information is available at the end of the article
} 


\section{Background}

An abnormal chromosome number (aneuploidy) is a feature of most solid tumors and is often accompanied by an elevated rate of chromosome mis-segregation termed chromosome instability (CIN) [1]. The gain or loss of entire chromosomes leads to large-scale changes in gene copy number and expression levels. The molecular mechanisms underlying CIN include defects in chromosome cohesion, mitotic checkpoint function, centrosome copy number, kinetochore-microtubule attachment dynamics, and cell-cycle regulation. While CIN can act as a driver of cancer genome evolution and tumor progression, recent findings point to the existence of a threshold level beyond which CIN becomes a barrier to tumor growth, and therefore, it can be exploited therapeutically. Janssen and co-authors [2] have analyzed the consequences of gradual increases in chromosome segregation errors on the viability of tumor cells and normal human fibroblasts. Partial reduction of essential mitotic checkpoint components in tumor cell lines caused mild chromosome mis-segregation, but no lethality. These cells were, however, much more sensitive to low doses of taxol, which enhances the amount and severity of chromosome segregation errors. Sensitization to taxol was achieved by reducing the levels of Mps1 or BubR1, proteins with dual roles in checkpoint activation and chromosome alignment. Importantly, untransformed human fibroblasts with reduced Mps1 levels could not be sensitized to sub-lethal doses of taxol. Thus, targeting the mitotic checkpoint and chromosome alignment simultaneously may selectively kill tumor cells. In another study [3], a set of genes was identified that are repressed in response to taxol treatment and over-expressed in tumors exhibiting CIN. The silencing of these genes caused cancer cell death, suggesting that these genes might be involved in the survival of aneuploid cells. In diploid cells, but not in chromosomally unstable cells, taxol causes the repression of CIN-survival genes, followed by cell death. Taking into account the fact that aneuploidization per se seems to be a very inefficient path towards cancer and additional hits are necessary for the generation of a cancer cell ([4] and references therein), these and other studies [5,6] indicate that increased destabilization of chromosomes might push genetically unstable cancer cells towards death, whereas more stable normal cells would be able to tolerate such insults.

Elevation of CIN as an approach to cancer therapy is attracting considerable attention [2-5]. However, none of the methods used to study CIN and its induction by environmental agents is entirely satisfactory. Karyotype analysis is bedeviled by the karyotypic variation already often present in cancer cell lines. Micronucleus assays $(\mathrm{MNi})$ are widely used to detect broken or lagging chromosomes, but fail to detect non-balanced chromosome segregation [7].
In this study, we developed a new assay for measuring CIN. This quantitative assay for chromosome mis-segregation is based on the use of the human artificial chromosome (HAC) constructed in our lab earlier as a gene therapy tool for the efficient and regulated expression of genes of interest [8-10]. The HAC contains centromeric repeats that form a functional centromere/kinetochore, allowing its stable inheritance as a nonessential chromosome, albeit with a loss rate roughly $10 \times$ that of the native chromosomes $[11,12]$. To adopt this HAC for CIN studies, an EGFP transgene was inserted into the HAC. This allowed the measurement of the HAC loss rate by routine flow cytometry. Thus, the HAC offers a sensitized and simple system to measure CIN, particularly after drug treatment. In this study, the HAC-based CIN assay has been verified using a set of well-known aneugens and clastogens. This new assay has the potential to be developed for high-through put screening methods to identify new compounds that elevate chromosome mis-segregation and drive lethal aneuploidy. New and potentially less toxic agents that selectively elevate CIN in cancer cells to promote cancer cell death identified with this new screening tool could lay the foundation for new treatment strategies for cancer.

\section{Methods \\ Cell lines}

Human fibrosarcoma HT1080 cells were cultured in Dulbecco's modified Eagle's medium (DMEM) (Invitrogen) supplemented with $10 \%(\mathrm{v} / \mathrm{v})$ tet system-approved fetal bovine serum (Clontech Laboratories, Inc.) at $37^{\circ} \mathrm{C}$ in $5 \%$ $\mathrm{CO}_{2}$. Hypoxanthine phosphoribosyltransferase (HPRT)deficient Chinese hamster ovary (CHO) cells (JCRB0218) carrying the alphoid ${ }^{\text {tetO }}$-HAC were maintained in Ham's F-12 nutrient mixture (Invitrogen) plus 10\% FBS with $8 \mu \mathrm{g} / \mathrm{ml}$ of BS (Funakoshi). After loading of the EGFP transgene cassette into the alphoid ${ }^{\text {tetO }}-\mathrm{HAC}$, the $\mathrm{CHO}$ cells were cultured in $1 \times$ HAT supplemented medium.

\section{Loading of the EGFP transgene cassette into the loxP site of alphoid ${ }^{\text {teto }}-\mathrm{HAC}$ in $\mathrm{CHO}$ cells}

A total of 3 to $5 \mu \mathrm{g}$ of a EGFP transgene plasmid (or X3.1-I-EGFP-I described previously [13]) and 1 to $2 \mu \mathrm{g}$ of the Cre expression $\mathrm{pCpG}-\mathrm{iCre}$ vector DNA were co-transformed into HPRT-deficient $\mathrm{CHO}$ cells containing the alphoid ${ }^{\text {tetO }}-\mathrm{HAC}$ by lipofection with FuGENERHD transfection reagent (Roche) or Lipofectamine 2000 (Invitrogen). HPRT-positive colonies were selected after 2 to 3 weeks growth in HAT medium. For each experiment, from 5 to 7 clones were usually selected. Correct loading of the EGFP transgene cassette into the HAC was confirmed by genomic PCR with a specific pair of primers that diagnose reconstitution of the HPRT gene [9]. 


\section{Microcell-mediated chromosome transfer}

The alphoid ${ }^{\text {tetO }}-$ HAC containing the EGFP transgene cassette (EGFP-HAC) was transferred from $\mathrm{CHO}$ cells to HT1080 cells using a standard microcell-mediated chromosome transfer (MMCT) protocol [13,14]. Blasticidin (BS) was used to select resistant colonies containing the HAC. Typically, three to ten $\mathrm{BS}^{\mathrm{R}}$ colonies were obtained in one MMCT experiment. $\mathrm{BS}^{\mathrm{R}}$ colonies were analyzed by FISH for the presence of the autonomous form of the HAC. The co-transfer of $\mathrm{CHO}$ chromosomes was examined using a sensitive PCR test for rodent-specific SINE elements [9].

\section{Flow cytometry}

Analysis of EGFP expression was performed on a FACS Calibur instrument (BD Biosciences) using CellQuest acquisition software and analyzed statistically with FlowJo software $[15,16]$. The cells were harvested by trypsin-treatment. Intensities of fluorescence were determined by flow cytometry. A minimum of $4 \times 10^{4}$ cells was analyzed for each cell sample.

\section{Drug treatment}

Nine different drugs were used in our experiments (Table 1). Our experiment protocol was as follows. HT1080 cells containing the EGFP-HAC were maintained on blasticidin selection to select for the presence of the HAC. Approximately $1 \times 10^{5}$ cells were cultured either in the presence or absence of blasticidin selection in parallel with a third culture that was exposed to the agent under examination to test its effect on EGFP-HAC segregation. The drug concentration applied was adjusted to the IC50 level for each compound which was determined using a proliferation assay described below. Concentrations of drugs and lengths

Table 1 Drugs used in this study

\begin{tabular}{|c|c|c|c|}
\hline Drug & Target & $\begin{array}{l}\text { Concentration/ } \\
\text { time treatment }\end{array}$ & $\begin{array}{l}\text { Fold increase of HAC } \\
\text { loss per cell division }\end{array}$ \\
\hline \multicolumn{4}{|c|}{ Microtubule-stabilizing drugs } \\
\hline$\overline{\text { Taxol }}$ & Beta-tubulin & 10 nM-overnight & $\times 47$ \\
\hline Ixabepilone & Beta-tubulin & 100 nM-overnight & $\times 31$ \\
\hline Docetaxel & Beta-tubulin & $10 \mathrm{nM}-2$ hrs & $\times 10$ \\
\hline Peloruside A & Beta-tubulin & 100 nM-overnight & $\times 32$ \\
\hline \multicolumn{4}{|c|}{ Microtubule-depolymerizing drugs } \\
\hline Nocodazole & Beta-tubulin & $1 \mu \mathrm{M}$-overnight & $\times 8$ \\
\hline \multicolumn{4}{|l|}{ Other drugs } \\
\hline SAHA & HDAC & $5 \mu \mathrm{M}$-overnight & $\times 1$ \\
\hline $\begin{array}{l}\text { VP16 } \\
\text { (etoposide) }\end{array}$ & TOP2 & $8 \mu \mathrm{M}-2 \mathrm{hrs}$ & $\times 7$ \\
\hline Reversine & $\begin{array}{l}\text { Aurora B, } \\
\text { MPS1 }\end{array}$ & $1 \mu \mathrm{M}-3$ days & $\times 14$ \\
\hline ZM-447439 & Aurora B & $1 \mu \mathrm{M}-3$ days & $\times 29$ \\
\hline
\end{tabular}

of treatment are presented in Table 1. Subsequently, the drug was removed by performing three consecutive medium washes and the cells were subsequently grown without blasticidin selection for 1-14 days. At the end of the experiment, cells were collected and analyzed by flow cytometry to detect the proportion of cells that retain EGFP fluorescence. This served as a measure of EGFP-HAC stability following drug treatment. For taxol and peloruside A, nine independent measuring of EGFP-HAC loss were carried out. The results were reproducible and the std were small (peloruside $\mathrm{A}: \mathrm{SD} \pm 0.9 \%$, taxol: $\mathrm{SD} \pm 1.1 \%$ ). Therefore, for other drugs, experiments were carried out in triplicate.

\section{Calculation of the rate of HAC loss after drug treatment}

The formula, $P_{n}=P_{0}(1-R)^{n}$ [17], routinely used to calculate the rate (R) of spontaneous HAC (or chromosome) loss, cannot be applied when cells are treated by a single dose of drug. So in our study, we first determined the normal rate of spontaneous HAC miss-segregation $\left(\mathrm{R}_{\text {Normal }}\right)$ in the host cell line HT1080 using the formula, $P_{\text {normal }}=P_{0}\left(\frac{2-R_{\text {Normal }}}{2}\right)^{n_{1}}$ (Figure 1$)$; where $\mathrm{P}_{0}$ is the percentage of $\operatorname{EGFP}(+)$ cells at the start of the experiment as determined by FACS. These cells were cultured under HAC selection conditions using blaticidin. $\mathrm{P}_{\text {Normal }}$ is the percentage $\operatorname{EGFP}(+)$ cells after culturing without HAC selection (no blasticidin) for a duration of $t_{1}$. In this study $t_{1}$ was 14 days. $\mathrm{n}_{1}$ is the number of cell doublings that occurs during culturing without blasticidin selection. The doubling time of HT1080 under normal growth conditions is approximately 18 hours. The number of cell divisions (n) is calculated by ( $\mathrm{t} /$ host cell doubling time).

Once $\left(\mathrm{R}_{\text {Normal }}\right)$ was obtained, the rate of HAC loss induced by drug treatment $\left(\mathrm{R}_{\text {Drug }}\right)$ is then determined using the formula, $P_{\text {Treated }}=P_{0}\left(\frac{2-R_{\text {Drug }}}{2}\right)^{n_{2}}\left(\frac{2-R_{\text {Normal }}}{2}\right)^{n_{3}}$. Justification of this algorithm is presented in Figure 1. As before, $\mathrm{P}_{0}$ represents the percentage of $\operatorname{EGFP}(+)$ cells at the start of the experiment, cultured under HAC selection condition. $\mathrm{P}_{\text {Treated }}$ is the percentage of EGFP $(+)$ cells at the end of a drug treatment experiment with a duration of $\left(t_{2}+t_{3}\right)$, where $t_{2}$ is the duration of drug treatment and $t_{3}$ is the duration of culturing after the drug is removed. $\left(t_{2}+t_{3}\right)$ was 14 days in this study. $n_{2}$ is the number of cell doublings that occurs during drug treatment, while $n_{3}$ is the number of cell doublings that occurs during the culturing without selection after drug treatment.

In the present study, the duration of most drug treatments were less than the duration of a single cell cycle of HT1080 $\left(t_{2}<18 \mathrm{hr}\right)$. We made the assumption that any significant increase in HAC loss occurs only during the first mitotic division after washing off a drug $\left(\mathrm{n}_{2}=1\right)$. Thus $n_{3}=(14 \mathrm{~d} / 18 \mathrm{hr}-1)$. The exceptions, reversine and 





(See figure on previous page.)

Figure 1 Calculation of the rate of HAC miss-segregation induced by drug treatment. Justification of the algorithm describing the dynamics of the accumulation of HAC-less cells caused by a single dose of chromosome-destabilizing compounds. This mathematical model assumes: 1) the drug kills cells non-selectively; 2) the drug's effect on HAC mis-segregation is not persistent and limited to the cell cycle when it is present; 3) spontaneous HAC loss after drug exposure does not change; 4) the HAC does not confer a selective advantage or disadvantage; 5) the cells are growing synchronously and 6) there is one HAC per cell. Assumptions 2) and 3) have been confirmed experimentally (see Additional file 1). (A) Our model assumes that when mis-segregation occurs during mitosis, one daughter cell will inherit a HAC while the other daughter cell does not. (B) Illustrated model of HAC lost in a population of cells. (C) Derivation of the general equation for HAC miss-segregation rate. $x$ is the number of cells which are EGFP(+); $y$ is the number of cell which are GFP(-); $\mathrm{R}$ is the probability of HAC miss-segregation; $n$ is the number of cell divisions; $P_{0}$ is the proportion of EGFP(+) cell at generation $F_{0} ; P_{1}$ is the proportion of EGFP(+) cell at generation $F_{1}$. (D) Calculation of rates (see Methods for more details).

ZM-447439 both severely inhibits cell growth, thus despite the 3 day drug treatment only one cell division is assumed to have occurred. We also assumed that HAC stability in subsequent cell divisions is no different from that of untreated cells.

The algorithm we used is valid between the ranges of 0 to 1 . $R$ values large than 1 indicate that the assumptions made in this model are incorrect. The assumption of synchronous growth in the model means that the estimated mis-segregation rate is lower than real values. As the spontaneous rate of HAC mis-segregation ( $\mathrm{R}_{\text {Normal }}$ ) was found to be low, this algorithm is relatively insensitive to the number of cell divisions that occurs post drug treatment. It is worth noting that the frequency of HAC loss in the clones carrying two copies of the HAC is indistinguishable from those containing a single copy of the HAC (data not shown). Therefore, the model is applicable when a cell inherits two copies of the HAC due to non-disjunction.

\section{Cell viability test}

MTS tetrazolium cell viability assays were done according to the manufacturer's instructions (CellTiter 96 AQueous Assay reagent; Promega). Briefly, the CellTiter 96 AQueous One Solution Reagent was added to each well and incubated at $37^{\circ} \mathrm{C}$ for $3 \mathrm{~h}$. Cell proliferation was determined by measuring the absorbance at $490 \mathrm{~nm}$ using a microtiter plate reader (Molecular Devices, Sunnyvale, CA). The half-maximal inhibitory concentration (IC50) was obtained from the MTS viability curves using GraphPad Prism 5. Experiments were carried out in triplicate.

\section{FISH analysis with PNA probes}

The presence of the HAC in an autonomous form was confirmed by FISH analysis as previously described $[8,9]$. HT1080 cells containing the HAC were grown in DMEM medium to $70-80 \%$ confluence. Metaphase cells were obtained by adding colcemid (Gibco) to a final concentration of $0.05 \mu \mathrm{g} / \mathrm{ml}$ and incubating overnight. Media was aspirated, and the plate washed with 1x PBS. Cells were removed from the plate by $0.25 \%$ Typsin, washed off with DMEM, pelleted and resuspended in 10 $\mathrm{ml}$ of $50 \mathrm{mM} \mathrm{KCl}$ hypotonic solution for $30 \mathrm{~min}$ at $37^{\circ} \mathrm{C}$. Cells were fixed by three washes of fixative solution $(75 \%$ acetic acid, 25\% methanol). Between each wash, cells were pelleted by centrifugation at $900 \mathrm{rpm}$ for $4 \mathrm{~min}$. Metaphase cells were evenly spread on a microscope slide and the fixative solution evaporated over boiling water. Dry slides were rehydrated with $1 \times$ PBS for $15 \mathrm{~min}$, and fixed in $4 \%$ formaldehyde- $1 \times \mathrm{PBS}$ for $2 \mathrm{~min}$, followed by three $5 \mathrm{~min} 1 \times$ PBS washes and ethanol series dehydration. PNA (peptide nucleic acid) labeled probes used were telomere (CCCTAA) ${ }_{3}-\mathrm{Cy} 3$ ) (PerSeptive Biosystems, Inc.) and tetO-alphoid array (FITC-OO-ACCACTCCCTATCAG) (Panagene, South Korea). Ten nanomol of each PNA probe was mixed with hybridization buffer and applied to the slide, followed by denaturation at $80^{\circ} \mathrm{C}$ for $3 \mathrm{~min}$. Slides were hybridized for 2 hours at room temperature in the dark. Slides were washed twice in $70 \%$ formamide, $10 \mathrm{mM}$ Tris $\mathrm{pH} 7.2,0.1 \% \mathrm{BSA}$ and followed by three washes with 1xTBS, $0.08 \%$ Tween-20. Slides were dehydrated gradually with a series of $70 \%, 90 \%$ and $100 \%$ ethanol washes and mounted (Vectorshield with DAPI). Images were captured using a Zeiss Microscope (Axiophot) equipped with a cooled-charge-coupled device (CCD) camera (Cool SNAP HQ, Photometric) and analyzed by IP lab software (Signal Analytics). The PNA-DNA hybrid probes demonstrated a high hybridization efficiency, staining intensity and adopt a stable duplex form with complementary nucleic acid.

\section{FISH analysis with the BAC probe}

HT1080 cells were processed for fluorescence in situ hybridization (FISH) after drug treatment followed by the 14 day washout. The probe used for FISH was BAC322-mer(tetO) DNA containing $40 \mathrm{~kb}$ of alphoid-tetO array cloned into a BAC vector as described previously [8]. Specifically, a BAC32-2-mer(tetO) clone contains an amplified synthetic alphoid DNA dimer. One monomer of this dimer is an alphoid DNA consensus sequence carrying the tetO sequence; another monomer is alphoid DNA from chromosome 17. This probe is specific to the HAC but also gives a low signal with centromeric regions of several endogenous chromosomes. BAC 
DNA was digoxigenin-labeled using a nick-translation kit with digoxigenin-11dUTP or biotin16-dUTP (Roche Diagnostics). Images were captured as before. The probe was denatured for $5 \mathrm{~min}$ at $95^{\circ} \mathrm{C}$ and added to the slides, which were incubated at $72^{\circ} \mathrm{C}$ for $2 \mathrm{~min}$ before overnight incubation at $39^{\circ} \mathrm{C}$. After washes with $0.1 \times \mathrm{SSC}$ at $65^{\circ} \mathrm{C}$ followed by a wash with $4 \times$ SSC $+0.1 \%$ Tween 20 at room temperature, standard procedures were used to detect biotinylated probes. Slides were mounted with VectaShield and screened for the presence or absence of the HAC. Between 70-250 metaphases were analyzed for each experiment (one drug treatment).

\section{Genomic DNA preparation and PCR analysis}

Genomic DNA for PCR analysis was prepared using a QIAmp DNA Mini Kit (QIAGEN Inc., Valencia, CA, USA). Reconstitution of the HPRT gene after Cre/lox-mediated recombination was determined by specific primers, Lox137-R 5' -agccttctgtacacatttcttctc3 ' and Rev \#65' -gctctactaagcagatggccacagaactag-3'. Cross contamination by hamster chromosomes was determined by specific primers detecting hamster SINEs: Cons B2 5'-ccatctgtaatgagatctgatgc-3', Ham B2-F 5'-gctc agaggttaagagcactgac-3' and Ham B2-R 5'-tgcttccat gtatatctgcacac- $3^{\prime}$. PCR products for sequencing were separated by agarose gel electrophoresis and gel extracted.

\section{Micronucleus formation assay (MNi)}

Duplicate cultures of cells were exposed to different compounds or DMSO control. Micronuclei formation was assessed by phase contrast microscopy in Table 2 after 20 hours. In the time course assay (Additional file 1), cells were harvested after $20 \mathrm{~h}$ treatment and grown in compound-free medium for $24 \mathrm{~h}, 5$ days and 10 days then placed on chamber slides. Dihydrocytochalasin B was added for $90 \mathrm{~min}$ to permit formation of cytokinesis blocked cells. Cells were washed with PBS and incubated with $0.075 \mathrm{M} \mathrm{KCl}$ then fixed in ice cold 3:1 v/v methanol: acetic acid and dried under vacuum. The frequency of micronucleated cells was

Table 2 Micronuclei (MNi) formation in the HAC-containing HT1080 cells treated by different drugs

\begin{tabular}{llll}
\hline Drug & \multicolumn{2}{l}{ Fraction of cells with MNi } & Mean \pm SD (\%) \\
\cline { 2 - 3 } & Exp. 1 & Exp. 2 & \\
\hline Vehicle $^{*}$ & $3 \%$ & $1 \%$ & $2 \pm 1$ \\
\hline 10 nM Taxol & $43 \%$ & $37 \%$ & $40 \pm 3$ \\
\hline 100 nM Peloruside A & $74 \%$ & $63 \%$ & $69 \pm 5$ \\
\hline $1 \mu$ M ZM447439 & $56 \%$ & $57 \%$ & $57 \pm 1$ \\
\hline 100 nM Nocodazole & $50 \%$ & $56 \%$ & $53 \pm 3$ \\
\hline
\end{tabular}

*Vehicle: $0.01 \%$ DMSO (this was the highest amount of DMSO used).

** Number of MN is given as means \pm SD in two independent experiments. assessed as described in [18] using DAPI staining and fluorescence microscopy.

\section{Results}

\section{Experimental design for identification of drugs that} elevate $\mathrm{CIN}$ in cancer cells

Figure 2 shows a general scheme of the new assay developed for measuring chromosome instability (CIN) based on the use of a human artificial chromosome (HAC) carrying the EGFP (enhanced green fluorescence protein) transgene. Due to the presence of a functional kinetochore, the EGFP-HAC is maintained as a non-essential 47th chromosome that replicates and segregates like a normal chromosome in human cells. Thus, the cells that inherit the HAC display green fluorescence, while cells that lack it do not. Normally, after growing in non-selective medium (i.e. in the absence of selection for the HAC), the majority of cells contain one copy of the HAC. After drug treatment, there are two possibilities: either no change in the EGFP level (no response to the drug) or an increased percentage of cells without HAC due to HAC segregation or replication errors (response to the drug treatment). It is expected that the control untreated cells should show uniform green fluorescence, while those that have lost HAC after drug treatment will exhibit reduced fluorescence that can be detected by flow cytometry.

\section{Construction of the HAC carrying a single copy of the EGFP transgene and its transfer to human cells}

The EGFP transgene cassette (X3.1-I-EGFP-I) was described previously [13]. To adapt the alphoid ${ }^{\text {tetO }}$-HAC [8] for CIN studies, the EGFP transgene cassette was inserted into a single loxP loading site of the HAC [13] in hamster HPRT-deficient Chinese Hamster Ovary ( $\mathrm{CHO}$ ) cells (Figure $3 \mathrm{~A}, \mathrm{~B}, 2 \mathrm{~B}$ ). In this cassette, EGFP is flanked by the $c H S 4$ insulator sequences to protect the transgene from epigenetic silencing. Targeting of the EGFP-cHS4 cassette into the loxP site was accompanied by reconstitution of the HPRT gene, allowing cell selection on HAT medium. Therefore, recombinant clones were selected by growth in HAT medium after two-three weeks. PCR analysis with specific primers (see Methods) confirmed that the HPRT gene was indeed reconstituted in all five drug-resistant clones analyzed (data not shown). The efficiency of cassette targeting into the HAC was $<1-3 \times 10^{-4}$. All of the $\mathrm{HPRT}^{+}$transfectants expressed the EGFP transgene, which was detected by fluorescence microscopy. FISH analysis of $\mathrm{CHO}$ metaphase spreads revealed the $\mathrm{HAC}$ in an autonomous form in four clones (data not shown). One clone containing the HAC was chosen for further experiments. 


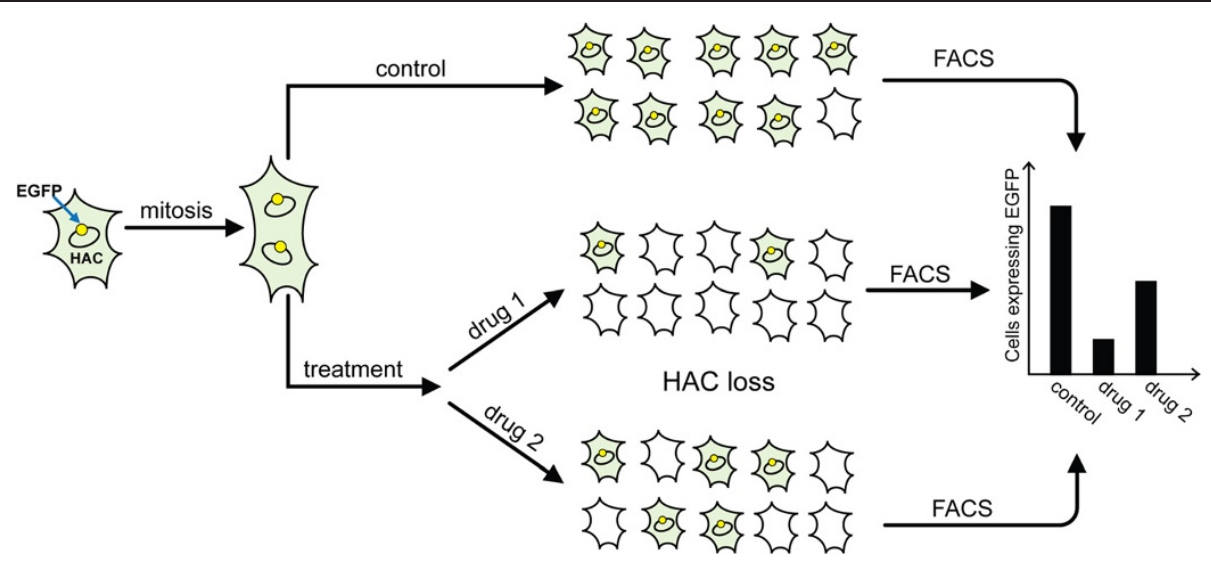

Figure 2 Scheme of an assay for measuring chromosome instability (CIN) based on the use of HAC containing the EGFP transgene. Cells that inherit the HAC display green fluorescence, while cells that lack it do not. It is expected that the control untreated cells should show uniform green fluorescence, while those that have lost HAC after drug treatment should be highly variable in fluorescence. Therefore, the actual number of cells with the EGFP-HAC can be measured by FACS. Thus, the compounds, which increase HAC loss and therefore increase spontaneous chromosome mis-segregation rates, may be identified.

The HAC containing the EGFP transgene was transferred from $\mathrm{CHO}$ to human HT1080 fibrosarcoma cells via microcell-mediated chromosome transfer (MMCT). Recipient cells were selected using the $B S$ resistance gene on alphoid ${ }^{\text {tetO }}$-HAC [8]. Ten BS-resistant clones that expressed the EGFP transgene were isolated from one MMCT experiment and analyzed using PCR with hamster-specific primers (see Methods) to rule out co-transfer of hamster chromosomes after MMCT. Fluorescence images of HT1080 cells carrying the HAC with the EGFP cassette are shown (Figure 3C). FISH analysis showed that the HAC was maintained autonomously without any detectable integration into the host genome in three out of five randomly selected clones. One clone (clone 12) containing an autonomously propagated EGFP-HAC was chosen for further analysis (Figure 3D). Based on the results from FISH analysis, the rate of spontaneous HAC loss was $7 \times 10^{-3}$ per cell division. The rate of HAC loss measured by the accumulation of non-fluorescent cells during growth in the absence of selection for the HAC by FACS was very similar, $13 \times 10^{-3}$ (Table 3 ), suggesting that non-fluorescent cells arise primarily through loss of the EGFP-marked HAC. Notably, mitotic stability of the EGFP-HAC is approximately 10 -fold less than stability of natural chromosomes in HT1080 cells $(\sim 1 \times$ $\left.10^{-3}\right)[11,12]$, making the system more sensitive for the detection of mis-segregation events. It is worth noting that in this clone, EGFP expression was stable for at least twelve months under selective conditions (data not shown). Based on these results, we conclude that the EGPF transgene on the HAC is stably expressed and that cells offer a sensitized system for analyzing chromosome loss.
Effect of aneugens and clastogens on the rate of HAC mis-segregation during mitotic divisions

We next investigated whether the EGFP/HAC-based assay could be used to detect compounds that cause chromosome loss and mis-segregation. HT1080 cells with an autonomously propagated EGFP-HAC were treated with eight known aneugens: taxol (pacilitaxel), docetaxel, peloruside A, ixabepilone, nocodazole, ZM447439, reversine, and SAHA (Table 1). Taxol, docetaxel, peloruside A, ixabepilone, and nocodazole are microtubule-targeting drugs [19]; ZM447439 and reversine are inhibitors of Aurora B and MPS1, respectively, and function in the spindle assembly checkpoint $[20,21]$. SAHA is an inhibitor of histone deacetylases (HDAC) [22]. Cells were also treated with the well-known clastogenic DNA topoisomerase II inhibitor VP16 (etoposide) [23].

For each compound, a cell cytotoxicity assay was carried out to determine IC50 values, i.e., the conditions under which the viability of cells would be around $50 \%$. We chose this parameter in order to normalize the results at the same percentage of viable cells. The evaluation of chromosome instability at the IC50 values has been used in many studies involving micronucleus scoring $[24,25]$. Time treatments and drug concentrations corresponding to IC50 are summarized in Table 1. For all compounds, the IC50 values were in the range clinically relevant concentrations, where applicable. After treatment, the cells were grown for two weeks in the absence of selection. Samples were analyzed every few days, and the proportion of non-fluorescent cells was determined. Non-fluorescent cells could be detected after a few days, and treated and untreated cell populations were clearly distinguishable after 5-7 days. The delay between HAC loss and the appearance of non-fluorescent cells is due to 


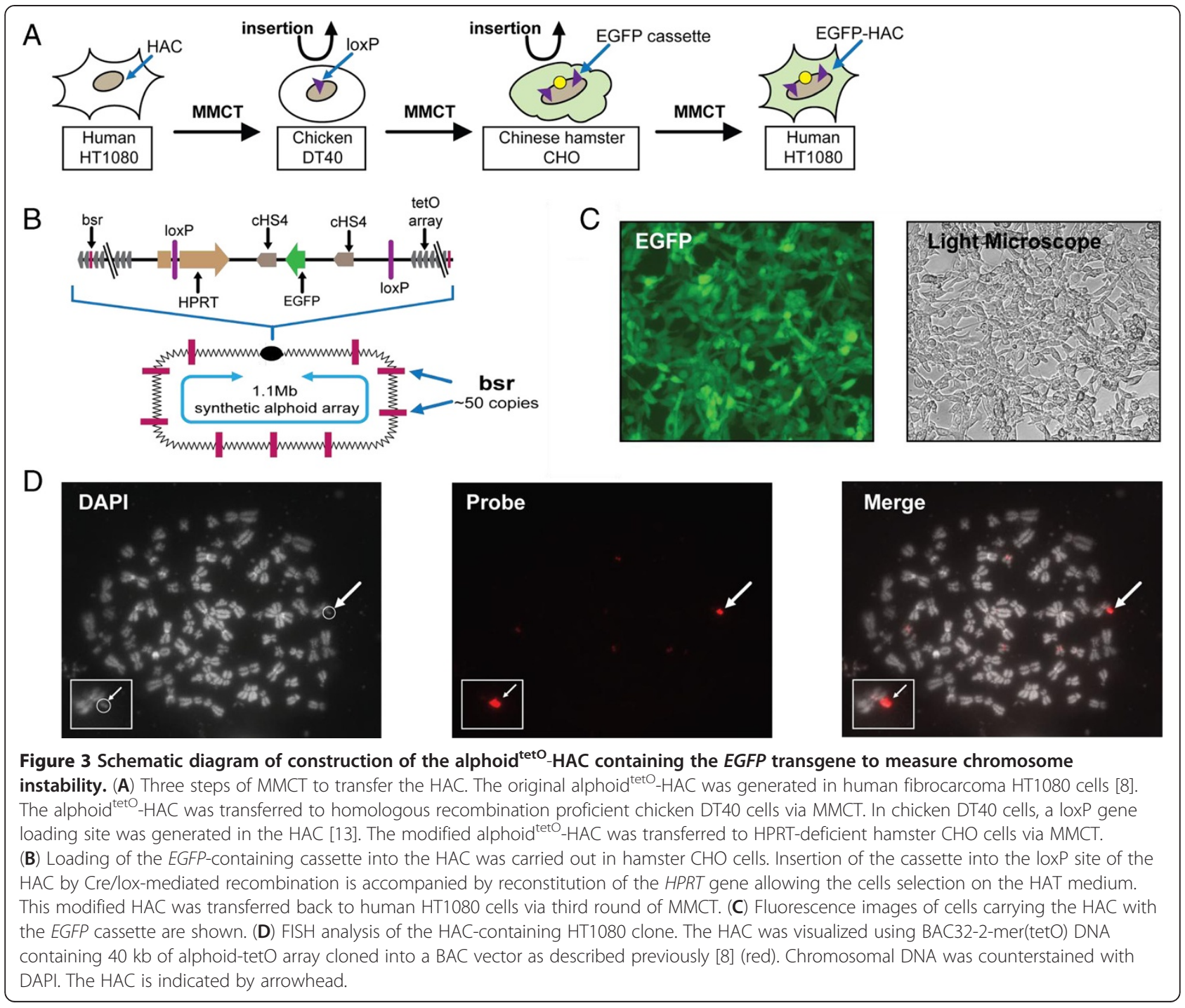

the persistence of the EGFP protein, which has a quite high half-life. Based on our analysis, sampling time has a broad interval (5-14 days) without a significant effect on the calculated rate of HAC loss (see Additional file 1). In most experiments, the cells were analyzed 14 days after drug washout. Figure 4 shows representative flow cytometry histograms illustrating loss of fluorescence for the cells treated by a single doze of taxol. It is important that the measuring is highly reproducible: The raw FACS data of three independent populations for drug treatments have standard deviations less than $1 \%$.

Figure 5 summarizes the estimated rates of HAC loss in response to different drugs. The rate of HAC loss $\left(\mathrm{R}_{\text {Drug }}\right)$ induced by drug treatment was calculated from the proportion of non-fluorescent cells in the population (Table 3) using the formula $P_{0}\left(\frac{2-R_{\text {Drug }}}{2}\right)^{n_{2}}\left(\frac{2-R_{\text {Normal }}}{2}\right)^{n_{3}}$ (see more details in Methods and Figure 1 legend).
As seen, a single dose of taxol greatly increases ( 50 times) the rate of HAC loss. Taxol (pacilitaxel) was isolated $\sim 40$ years ago and is currently administered in a large variety of indications, including solid tumors and haematological malignancies [19] and references therein]. While its mechanism of action remains controversial, accumulating data suggest that at clinically relevant concentrations, taxolmediated cell death involves elevation of chromosome missegregation that is incompatible with cancer cell survival [26] and references therein]. HAC loss was also significantly increased by peloruside $\mathrm{A}$, ixabepilone, which also binds to the microtubule as taxol, and by the Aurora B inhibitor ZM-447439. For the other analyzed compounds except SAHA, the rates of HAC loss were also increased compared to controls, but much lower compared to those obtained after taxol or peloruside A treatment. The lowest increase of HAC loss was obtained after treatment by the inhibitor of histone deacetylases, SAHA. 
Table 3 Comparison between FISH and FACS data to evaluate HAC induced by drug treatment

\begin{tabular}{llll}
\hline Drug & $\begin{array}{l}\text { FISH } \\
\text { \% Cells } \\
\text { with HAC }\end{array}$ & $\begin{array}{l}\text { FACS } \\
\text { \% Cells } \\
\text { with EGFP }\end{array}$ & $\begin{array}{l}\text { Rate of HAC loss } \\
\text { per cell division** }\end{array}$ \\
\hline No drug & $86(100)^{*}$ & $85.1 \pm 0.9$ & $13 \times 10^{-3}$ \\
\hline Taxol & $59(139)$ & $59.5 \pm 0.9$ & $597 \times 10^{-3}$ \\
\hline Ixabepilone & $71(116)$ & $68.0 \pm 0.1$ & $396 \times 10^{-3}$ \\
\hline Docetaxel & $74(101)$ & $79.1 \pm 1.8$ & $133 \times 10^{-3}$ \\
\hline Peloruside A & $68(71)$ & $67.4 \pm 0.9$ & $411 \times 10^{-3}$ \\
\hline Nocodazole & $79(77)$ & $80.3 \pm 0.9$ & $107 \times 10^{-3}$ \\
\hline SAHA & $79(160)$ & $84.3 \pm 1.2$ & $12 \times 10^{-3}$ \\
\hline VP16 (Etoposide) & $86(156)$ & $80.7 \pm 0.9$ & $96 \times 10^{-3}$ \\
\hline Reversine*** & $77(71)$ & $79.0 \pm 0.5$ & $176 \times 10^{-3}$ \\
\hline ZM-447439*** & $75(75)$ & $70.4 \pm 0.5$ & $374 \times 10^{-3}$
\end{tabular}

* In parentheses, the number of metaphases screened for the presence or absence of the HAC.

** Rates of the HAC loss after drug treatment were calculated from FACS data using the formula $P_{\text {Treated }}=P_{0}\left(\frac{2-R_{\text {Drug }}}{2}\right)^{n_{2}}\left(\frac{2-R_{\text {Normal }}}{2}\right)^{n_{3}}$.

*** A real rate of HAC loss may be a little bit lower after treatment by inhibitors of Aurora B and MPS1 because reversine and ZM-447439 do not completely block cell divisions.

In separate experiments, the relationship of HAC loss to chemical dose was investigated. Treatment of cells with higher doses of the compounds kills more cells but does not increase the rate of HAC loss. This may be explained if cells arrest, then apoptosis in response to damage of the spindle or if the daughter cells are not viable. We also performed the experiments with lower doses of drugs, i.e. with concentrations $1 / 2,1 / 4,1 / 8$ and $1 / 16$ of the concentration corresponding to IC50. For some drugs, the frequency of HAC loss was dose independent while for others displayed a linear dose-dependence. For example, HAC loss was decreased in an almost linear fashion after treatment by VP16 (which is inhibitor of TOP2) with lower doses. In contrast, our analysis revealed a non-linear dose-dependent decrease in HAC loss frequencies for nocodazole and taxol, which were previously reported as compounds that interact with the mitotic spindle (thresholded concentration-effect response) [27].

FISH analysis was used to confirm that the appearance of non-fluorescent cells detected by flow cytometry corresponds to HAC loss events and to exclude possible alternative explanations such as mutations in the EGFP gene or its epigenetic silencing. Quantitative analysis of metaphase chromosome spreads using a HAC-specific probe (see Methods) correlated with the data on HAC loss determined by FACS (Table 3). Therefore, we conclude that the appearance of non-fluorescent cells is caused by HAC loss.

An unexpected result was the different effects of microtubule-binding drugs on HAC stability. Ixabepilone, docetaxel, and peloruside A are microtubule-stabilizing agents similar to taxol [19]. However, each drug exhibited a different effect on HAC mitotic stability, suggesting a different cell response to these compounds. Interestingly, under these conditions, all of the analyzed drugs induced micronuclei formation in the HAC-containing HT1080 cells (Table 2) similar to that reported for other cell lines [27]. However, there was no detectable correlation between frequencies of $\mathrm{MNi}$ formation (that varied between $37 \%$ and $63 \%$ ) and rates of HAC loss determined by FISH and FACS (Table 3). Thus, aneugens with a similar mechanism of action and cytotoxicity may differ from each other by their effect on the mitotic stability of the non-essential human artificial chromosome. It is
A

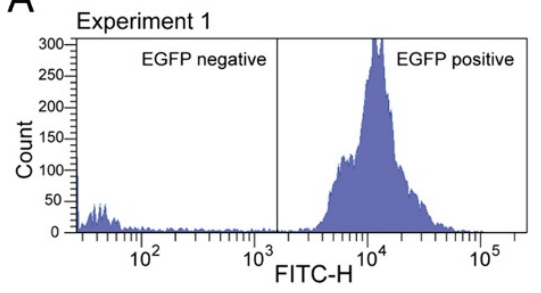

B Experiment 1

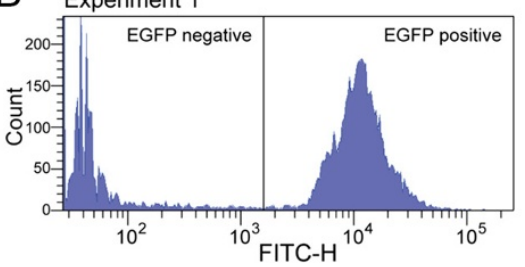

No Drug

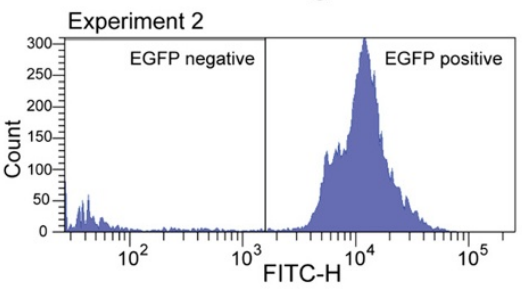

Taxol 10nM



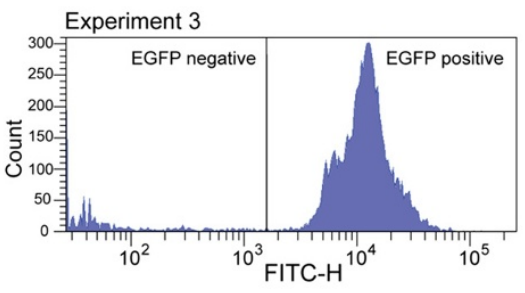

Experiment 3

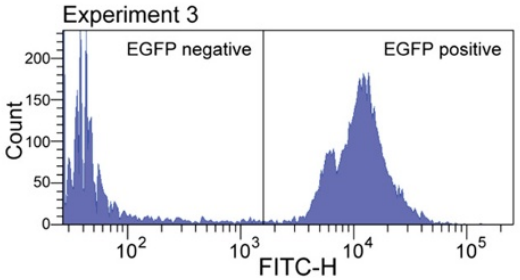

Figure 4 Two flow cytometry histograms illustrating mitotic stability of the EGFP-HAC in HT1080 cells (A) before and (B) after treatment by taxol. The $x$-axis represents the intensity of the fluorescence, the $y$-axis the number of cells. The bar stands for the amount of positive cells. The results of triplicate experiments are shown. 


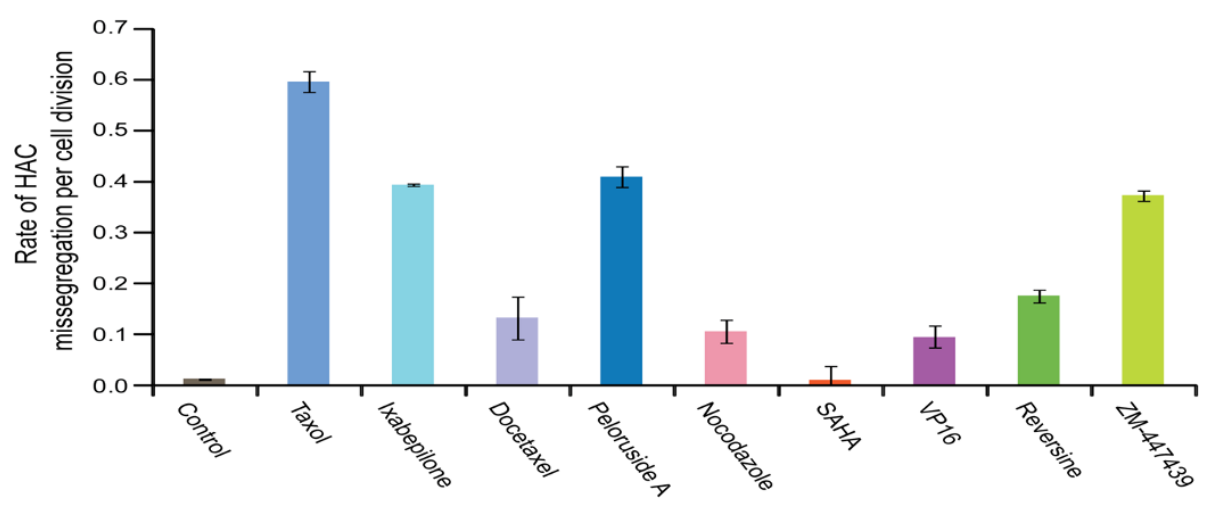

Figure 5 Mitotic stability of the EGFP-HAC in HT1080 human cells treated by nine different drugs. A rate of HAC loss per generation was calculated as described in Methods and Figure 1. Blue bars correspond to the frequency of HAC loss when the cells were treated by taxol and its derivatives. The control corresponds to a frequency of spontaneous loss of the EGFP-HAC in human HT1080 cells.

unlikely that karyotyping or another variant of MNi tests can detect such differences between the compounds. Based on these results, we conclude that a newly developed EGFP/HAC-based assay for measuring CIN is a more sensitized system than other previously described methods to detect chromosome mis-segregation.

\section{Discussion}

Targeting of CIN in cancer therapy requires measurement of the accuracy of chromosome transmission. At present, a variety of methods is used to study chromosome instability (CIN) and its induction by environmental agents $[7,28]$ and references therein]. Because test systems screening for numerical chromosomal effects rely on labor-intensive microscopic assessment, the micronucleus (MNi) formation test is the most widely used method for large-scale detection of broken or lagging chromosomes [7]. However, because the origins and fates of $\mathrm{MNi}$ have not been completely elucidated [29,30], intra- and inter-laboratory variability in scoring is still common [31], and complicates the development of a standard protocol for quantitative measurement of chromosome loss rates based on the appearance of $\mathrm{MNi}$. It is also noteworthy that the $\mathrm{MNi}$ assay does not measure the fraction of drug-arrested cells that undergo mitosis and form viable aneuploid cells.

This work describes a new assay for measuring CIN in response to drug treatment that overcomes the limitations of current approaches. The assay is based on quantitative measurements of mitotic loss rates of a nonessential human artificial chromosome (HAC) carrying a transgene encoding the green fluorescent protein (EGFP). Thus, cells that inherit the HAC fluoresce green, while cells that lack it do not. The proportion of cells that lose the HAC in response to drugs is measured using FACS, which allows the objective and accurate assessment of chromosome loss in a large number of cells (e.g., $10^{6}$ ) within minutes. Because only viable cells are studied, the long-term effects of aneugen exposure on chromosome loss are readily measured. It is worth noting that a similar approach but involving natural chromosome was previously attempted [32], but proved not to be useful for CIN studies, as the rates of chromosome loss were too low to be measured accurately. In the present study, the use of a HAC reporter that is sensitized for chromosome loss circumvents this problem. The HAC contains a functional kinetochore and its behavior during mitotic divisions does not differ from that of normal chromosomes $[8,10]$, suggesting that destabilization of natural chromosomes in response to drug treatment will be increased proportionally to that observed for the HAC.

As proof of principle, the EGFP/HAC-based assay was applied to analyze a set of well-known anti-mitotic, spindle-targeting compounds previously reported to induce micronucleus formation and chromosome loss. For each drug, the rate of HAC loss was measured by flow cytometry and confirmed by FISH analysis. The most interesting result obtained from these experiments is the observation that despite their similar cytotoxicity, the analyzed drugs affect the rates of HAC mis-segregation during mitotic divisions differently. Moreover, we found that aneugens with proposed similar mechanisms of action and cytotoxicity may greatly differ from each other by their effect on the mitotic stability of the non-essential human artificial chromosome. Thus, the assay described here is sensitive enough to discriminate between drugs with similar mechanisms of action in order to reveal those with the highest activity on chromosome stability. In future drug screening studies, taxol exhibiting the highest effect on HAC stability may be used as a positive control.

In this work to compare different compounds, the cells were analyzed 14 days after drug washout. At the same time, the sampling time for HT1080 cells has a broad 
interval and fluorescence of the treated cells can be measured even after 5-7 days without a significant effect on the calculated rate of HAC loss (see Additional file 1). For other cell lines, where the EGFP protein is more stable, the sampling time may be exceeded up to 10-14 days. However, the speed of the assay can be significantly increased using a modified EGFP with a reduced half-life (e.g., EGFP with a degron box sequence) that would convert an assay into high-throughput screening. The transfer of EGFP-HAC into other cell lines will make possible to assess effects of inhibitors in different genetic backgrounds such as cell lines harboring mutations in genes that regulate the spindle assembly checkpoint. It is worth noting that recent innovations have allowed the generation of HACs with the EGFP transgene de novo in a broad variety of human cell lines [33].

This new HAC-based flow cytometry assay may be also applied for identifying genes controlling chromosome segregation in human cells because it is more sensitive to perturbations of spindle assembly and cell cycle progression than assays based on the analysis of stability of natural chromosomes [34]. Earlier, the development of conceptually simple color colony assays in yeast provided a powerful genetic tool to assess the rates of chromosome mis-segregation and to identify mutants deficient in this process [35]. Similarly, in humans, the EGFP/HAC-based chromosome loss assay may be used to reveal new human genes involved in chromosome transmission.

\section{Conclusions}

We have developed a new assay for measuring chromosome instability based on the use of a non-essential human artificial chromosome (HAC) carrying a constitutively expressed EGFP transgene. This simple assay allows for a quick and efficient screen of hundreds of drugs to identify those affecting chromosome mis-segregation. It also allows ranking of compounds with the same or similar mechanism of action based on their effect on the rate of chromosome loss.

\section{Additional file}

Additional file 1: Figure S1. (A) Micronuclei (MNi) formation (indicated by arrows) in the HAC-containing HT1080 cells treated by drugs. Scale bar $=10 \mu \mathrm{M}$. (B) The kinetics of MNi formation after drug treatment at different time points after washout. The experiment was performed for taxol $(10 \mathrm{nM})$ and nocodazole $(1 \mu \mathrm{M})$. (C) Rates of HAC loss calculated using FACS profiles of cells before, after drug treatment and at different time points after washout. The experiment was performed for taxol and ixabepilone.

\section{Competing interests}

The authors declare they have no competing interests.

\section{Authors' contributions}

HSL, NCOL, BRG, AS, AVK performed the experiments. RB assisted with experiments. BRG, HM, WCE, NK, VL analyzed the data. VL designed the study. VL, NK, WCE, BRG contributed to writing the manuscript. All authors read and approved the final manuscript.

\section{Acknowledgements}

This work was supported by the Intramural Research Program of the $\mathrm{NIH}$, National Cancer Institute, Center for Cancer Research, USA (V.L.), a Wellcome Trust Principal Research Fellowship [grant number 073915] (W.C.E.), the Grand-in-Aid for Scientific Research from Ministry of Education, Culture, Sports, Science and Technology of Japan (H.M.) and the Kazusa DNA Research Institute Foundation (H.M.) and the Indiana Genomics Initiative (INGEN) (B.R.G.). INGEN is supported in part by the Lilly Endowment. Reversine and ZM-447439 were kindly provided by Dr. Alexey Arnautov (National Institute of Child Health and Human Development/ NICHHD, NIH). Ixabepilone and docetaxel were obtained from Dr. Marianne Poruchynsky (National Cancer Institute/NCl, NIH). Peloruside A was kindly provided by Dr. Dan Sackett (National Institute of Child Health and Human Development/ $\mathrm{NICHHD}, \mathrm{NIH}$ ). The authors also would like to thank the CRC, LRBGE Fluorescence Imaging Facility $(\mathrm{NIH})$ and personally Drs. Karpova and Dr. McNally for instructions, consultations and help with the usage of a DeltaVision microscopy imaging system. We also would like to thank Dr. Elisabetta Leo for help in some experiments.

\section{Author details}

${ }^{1}$ Laboratory of Molecular Pharmacology, National Cancer Institute, Bethesda, MD 20892, USA. ²Department of Medical and Molecular Genetics, Indiana University School of Medicine, Indiana University Melvin and Bren Simon Cancer Center, Indianapolis, IN 46202, USA. 'aboratory of Cell Engineering, Department of Human Genome Research, Kazusa DNA Research Institute, 2-6-7 Kazusa-Kamatari, Kisarazu, Chiba 292-0818, Japan. ${ }^{4}$ Wellcome Trust Centre for Cell Biology, University of Edinburgh, Edinburgh EH9 3JR, Scotland.

Received: 14 January 2013 Accepted: 14 May 2013

Published: 22 May 2013

\section{References}

1. Thompson SL, Bakhoum SF, Compton DA: Mechanisms of chromosomal instability. Curr Biol 2010, 20:R285-R295.

2. Janssen A, Kops GJ, Medema RH: Elevating the frequency of chromosome mis-segregation as a strategy to kill tumor cells. Proc Natl Acad Sci USA 2009, 106:19108-19113.

3. Swanton C, Nicke B, Schuett M, Eklund AC, Ng C, Li Q, Hardcastle T, Lee A, Roy R, East P, Kschischo M, Endesfelder D, Wylie P, Kim SN, Chen JG, Howell M, Ried T, Habermann JK, Auer G, Brenton JD, Szallasi Z, Downward J: Chromosomal instability determines taxane response. Proc Natl Acad SCi USA 2009, 106:8671-8676.

4. Colombo R, Moll J: Targeting aneuploid cancer cells. Expert Opin Ther Targets 2011, 15:595-608.

5. Janssen A, Kops GJ, Medema RH: Targeting the mitotic checkpoint to kill tumor cells. Hormones \& Cancer 2011, 2:113-116.

6. Janssen A, Van der Burg M, Szuhai K, Kops GJ, Medema RH: Chromosome segregation errors as a cause of DNA damage and structural chromosome aberrations. Science 2011, 333:1895-1898.

7. Kirsch-Volders M, Elhajouji A, Cundari E, Van Hummelen P: The in vitro micronucleus test: a multi-endpoint assay to detect simultaneously mitotic delay, apoptosis, chromosome breakage, chromosome loss and non-disjunction. Mutat Res 1997, 392:19-30.

8. Nakano M, Cardinale S, Noskov VN, Gassmann R, Vagnarelli P, Kandels-Lewis S, Larionov V, Earnshaw WC, Masumoto H: Inactivation of a human kinetochore by specific targeting of chromatin modifiers. Dev Cell 2008, 14:507-522

9. Kim JH, Kononenko A, Erliandri I, Kim TA, Nakano M, lida Y, Barrett JC, Oshimura M, Masumoto H, Earnshaw WC, Larionov V, Kouprina N: Human artificial chromosome (HAC) vector with a conditional centromere for correction of genetic deficiencies in human cells. Proc Natl Acad Sci USA 2011, 8:20048-20053.

10. Kouprina N, Earnshaw WC, Masumoto H, Larionov V: A new generation of human artificial chromosomes for functional genomics and gene therapy. Cell Mol Life Sci 2012, 70:1135-1148. 
11. Rudd MK, Mays RW, Schwartz S, Willard HF: Human artificial chromosomes with alpha satellite-based de novo centromeres show increased frequency of nondisjunction and anaphase lag. Mol Cell Biol 2003, 23:7689-7697.

12. Spence JM, Mills W, Mann K, Huxley C, Farr CJ: Increased missegregation and chromosome loss with decreasing chromosome size in vertebrate cells. Chromosoma 2006, 115:60-74

13. lida Y, Kim JH, Kazuki Y, Hoshiya H, Takiguchi M, Hayashi M, Erliandri I, Lee HS, Samoshkin A, Masumoto H, Earnshaw WC, Kouprina N, Larionov V, Oshimura M: Human artificial chromosome with a conditional centromere for gene delivery and gene expression. DNA Res 2010, 17:293-301

14. Koi M, Shimizu M, Morita H, Yamada H, Oshimura M: Construction of mouse A9 clones containing a single human chromosome tagged with neomycin-resistance gene via microcell fusion. Jph J Cancer Res 1989, 80:413-418.

15. Kim JH, Ebersole T, Kouprina N, Noskov VN, Ohzeki Jl, Masumoto H, Mravinac B, Sullivan BA, Dovat S, Pavlicek A, Pack SD, Kwon YW, Flanagan $P T$, Loukinov D, Lobanenkov V, Larionov V: Human pericentromeric gamma-satellite DNA maintains open chromatin structure and protects a transgene from epigenetic silencing at an ectopic site. Genome Res 2009, 19:533-544.

16. Ebersole T, Kim JH, Samoshkin A, Kouprina N, Pavlicek A, White R, Larionov $\checkmark$ : tRNA genes protect a reporter gene from epigenetic silencing in mouse cells. Cell Cycle 2011, 10:2729-2791.

17. Ikeno M, Grimes B, Okazaki T, Nakano M, Saitoh K, Hoshino H, McGill NI, Cooke $\mathrm{H}$, Masumoto $\mathrm{H}$ : Construction of YAC-based mammalian artificial chromosomes. Nat Biotechnol 1998, 16:431-439.

18. Fenech M: Cytokinesis-block micronucleus cytome assay. Nat Protoc 2007, 2:1084-1104.

19. Dumontet $C$, Jordan MA: Microtubule-binding agents: a dynamic field of cancer therapeutics. Nat Rev Drug Discov 2010, 9:790-803.

20. Georgieva I, Koychev D, Wang Y, Holstein J, Hopfenmüller W, Zeitz M, Grabowski P: ZM447439, a novel promising aurora kinase inhibitor, provokes antiproliferative and proapoptotic effects alone and in combination with bio- and chemotherapeutic agents in gastroenteropancreatic neuroendocrine tumor cell lines. Neuroendocrinology 2010, 91:121-130.

21. Santaguida S, Tighe A, D'Alise AM, Taylor SS, Musacchio A: Dissecting the role of MPS1 in chromosome biorientation and the spindle checkpoint through the small molecule inhibitor reversine. J Cell Biol 2010, 190:73-87.

22. Robbins AR, Jablonski SA, Yen TJ, Yoda K, Robey R, Bates SE, Sackett DL: Inhibitors of histone deacetylases alter kinetochore assembly by disrupting pericentromeric heterochromatin. Cell Cycle 2005, 4:717-726.

23. Pommier $Y$, Leo E, Zhang H, Marchand C: DNA topoisomerases and their poisoning by anticancer and antibacterial drugs. Chem Biol 2010, 17:421-433

24. Kirkland D, Pfuhler S, Tweats D, Aardema M, Corvi R, Darroudi F, Elhajouji A, Glatt H, Hastwell P, Hayashi M, Kasper P, Kirchner S, Lynch A, Marzin D, Maurici D, Meunier JR, Müller L, Nohynek G, Parry J, Parry E, Thybaud V, Tice $R$, Van Benthem J, Vanparys $P$, White $P$ : How to reduce false positive results when undertaking in vitro genotoxicity testing and thus avoid unnecessary follow-up animal tests: Report of an ECVAM Workshop. Mutat Res 2007, 628:31-55.

25. Hashimoto K, Nakajima Y, Matsumura S, Chatani F: An in vitro micronucleus assay with size-classified micronucleus counting to discriminate aneugens from clastogens. Toxicol In Vitro 2010, 24:208-216.

26. Ingemarsdotter CK, Baird SK, Connell CM, Öberg D, Halldén G, McNeish IA Low-dose paclitaxel synergizes with oncolytic adenoviruses via mitotic slippage and apoptosis in ovarian cancer. Oncogene 2010, 29:6051-6063.

27. Elhajouji A, Lukamowicz M, Cammerer Z, Kirsch-Volders M: Potential thresholds for genotoxic effects by micronucleus scoring. Mutagenesis 2011, 26:199-204.

28. Thompson SL, Compton DA: Examining the link between chromosome instability and aneuploidy in human cells. J Cell Biol 2008, 180:665-672.

29. Asur RS, Thomas RA, Tucker JD: Chemical induction of the bystander effect in normal human lymphoblastoid cells. Mutat Res 2009, 676:11-16.

30. Meintières S, Marzin D: Apoptosis may contribute to false-positive results in the in vitro micronucleus test performed in extreme osmolality, ionic strength and $\mathrm{pH}$ conditions. Mutat Res 2004, 560:101-118.
31. Crasta K, Ganem NJ, Dagher R, Lantermann AB, Ivanova EV, Pan Y, Nezi L, Protopopov A, Chowdhury D, Pellman D: DNA breaks and chromosome pulverization from errors in mitosis. Nature 2012, 482:53-58.

32. Burns EM, Christopoulou L, Corish P, Tyler-Smith C: Quantitative measurement of mammalian chromosome mitotic loss rates using the green fluorescent protein. J Cell Sci 1999, 112:2705-2714.

33. Ohzeki Jl, Bergmann JH, Kouprina N, Noskov VN, Nakano M, Kimura H, Earnshaw WC, Larionov V, Masumoto H: Breaking the HAC Barrier: Histone H3K9 acetyl/methyl balance regulates CENP-A assembly. EMBO J 2012, 31:2391-2402.

34. Thompson SL, Compton DA: Chromosome missegregation in human cells arises through specific types of kinetochore-microtubule attachment errors. Proc Natl Acad Sci USA 2011, 108:17974-17978.

35. Spencer F, Gerring SL, Connelly C, Hieter P: Mitotic chromosome transmission fidelity mutants in Saccharomyces cerevisiae. Genetics 1990, 124:237-249.

doi:10.1186/1471-2407-13-252

Cite this article as: Lee et al:: A new assay for measuring chromosome instability (CIN) and identification of drugs that elevate CIN in cancer cells. BMC Cancer 2013 13:252.

\section{Submit your next manuscript to BioMed Central and take full advantage of:}

- Convenient online submission

- Thorough peer review

- No space constraints or color figure charges

- Immediate publication on acceptance

- Inclusion in PubMed, CAS, Scopus and Google Scholar

- Research which is freely available for redistribution

Submit your manuscript at www.biomedcentral.com/submit
C) Biomed Central 